\title{
Implementing the eRobotics Approach by Combining Advanced Rendering Techniques and Complex Simulations in a Modern, Multi-domain VR Simulation System
}

\author{
Nico Hempe and Jürgen Roßmann
}

\begin{abstract}
Multi-domain VR simulation systems provide a framework to bring together various modules in order to fulfill all desired tasks; however, rendering capabilities have mostly been neglected in these systems due to economic aspects or technical limitations. In this contribution, we will present the concepts of eRobotics, which have been motivated by the demand for a holistic and comprehensive multi-purpose tool for the application in a wide range of domains dealing with robotics. We will show how realistic virtual environments as well as the close interplay between rendering and simulation modules can bring simulation results closer to those achieved in real world setups. In particular, the simulation of various optical sensors like digital camera systems, laser range scanners (LiDAR) and time-of-flight (ToF) cameras can benefit from these developments to further shift costly and time-consuming physical prototypes to the end of the development process.
\end{abstract}

Index Terms-VR simulation system, sensor simulation, real-time computer graphics, eRobotics.

\section{INTRODUCTION}

Today, Virtual Reality (VR) simulation technology is a well-known field of engineering and widely applied in research and in the industry. In particular, Virtual Prototyping (VP) has become an important part in robotics-related engineering and development. By integrating further system components and simulating their collaboration, Virtual Prototypes can become virtual testbeds (VT), which allow for the testing of the system as a whole, including all related technical components [1]. As illustrated in Fig. 1, sensors are an essential part of almost any domain dealing with robotics, ranging from industrial robotics over mobile robotics up to space robotics; hence, current robot simulators, such as USARSim [2] or Player/Stage/Gazebo [3], provide different sensor simulations. A realistic sensor simulation that matches real world results is vital to improve the robustness of the developed software components before testing them in costly hardware setups. Finally, sensor data from synthetic environments is extremely important for the evaluation and the comparison of sensor-related algorithms, since a simulation system also provides the corresponding ground truth that is necessary for tuning and validating of

Manuscript received March 25, 2015; revised July 13, 2015. The work presented in this paper was done as part of the Virtual Forest project. The Virtual Forest project is co-financed by the European Union and the federal state of North Rhine- Westphalia, European Regional Development Fund (ERDF). Europe - Investing in our future.

The authors are with Institute for Man-Machine Interaction at the RWTH Aachen University, Aachen, Germany (e-mail: hempe@mmi.rwth-aachen.de, rossmann@mmi.rwth-aachen.de). sensor-related systems.

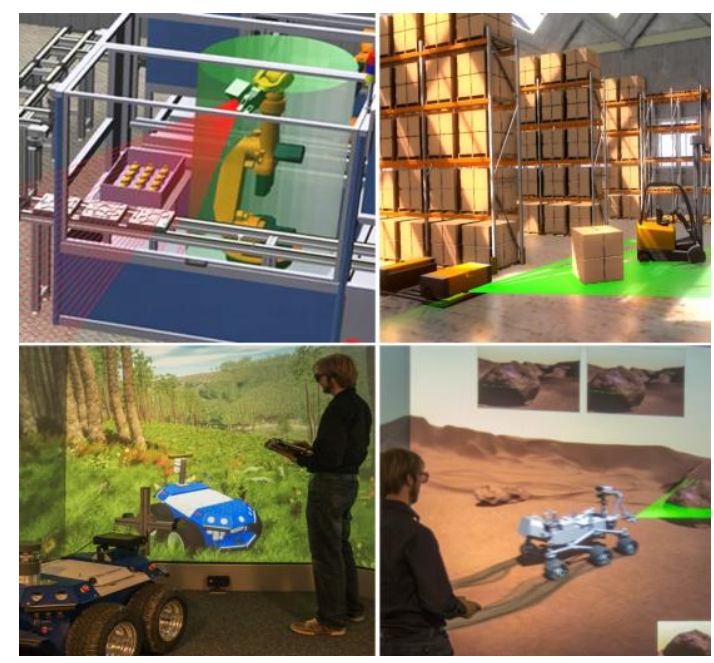

Fig. 1. The realistic simulation of various sensors in vital in many domains that address robotic domains.

Simulations get more and more complex and the accuracy requirements rise with advanced technology development; hence, the calculation power of modern graphics hardware must not be neglected for simulation purposes. Apart from the current trend of general-purpose computing on graphics processing units (GPGPU) [4], recent publications showed that rendering approaches can be applied to realize real-time simulations of digital camera systems [5], LiDAR scanners [6] and ToF cameras [7]. Synergy effects, which arise from the combination of simulation and rendering technologies in a holistic eRobotics system, can help to face the complexity issue and similarly increase accuracy and performance of optical sensor simulations significantly.

\section{THE EROBOTICS APPROACH}

The research field of eRobotics can be understood as an intuitively applicable eService using VR technologies in advanced robotic applications and in engineering [8]. As illustrated in Fig. 2, eRobotics applications cover all aspects of VR. On the one hand, attractive virtual environments and visualizations help to understand complex mechanisms and correlations, in particular when presenting them to people not so deeply involved in the specific area of expertise. On the other hand, eRobotics systems are complex enough to be used as a full-featured engineering tool, which can be used in any phase of the development process right from the beginning. 


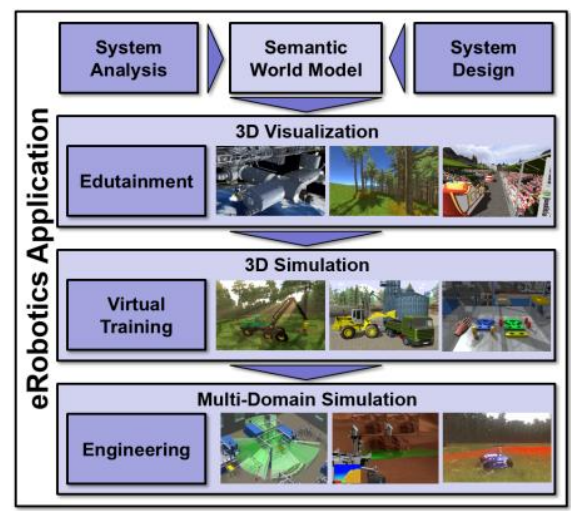

Fig. 2. eRobotics applications cover all aspects of VR. Model descriptions are based on semantic world models.

These systems can act as decision support systems (DSS) in early concept phase to make fundamental design decisions when evaluating technical possibilities. Which sensors suit best to the developed system? Which measurement errors are acceptable in order to achieve satisfactory results? What degree of error can be tolerated before the results start to worsen? To answer these questions, eRobotics applications provide engineers with a tool to dynamically alter various components or simulation parameters and directly experience the results in realistic real-time $3 \mathrm{D}$ environments. In order to fulfill the eRobotics concepts considering simulation and rendering tasks for a broad range of different domains, novel concepts and system structures for the underlying database, simulation and rendering technology were necessary.

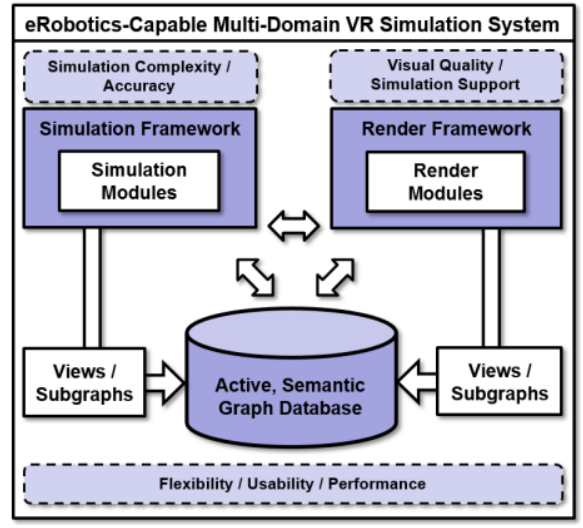

Fig. 3. General structure of the system, which allows for the integration and intercommunication of simulation- and rendering-related modules.

As illustrated in Fig. 3, the key idea of eRobotics is to base simulation and rendering components on a central, active and extendible object-oriented graph database to overcome technical limitations when trying to combine the advantages of rendering and simulation tasks in a holistic multi-domain VR simulation system. Each module can define its own view to the database and integrate subgraphs to meet its specific data structure requirements. As shown in recent publications, this flexible database in combination with a micro-kernel-based system design enable to realize a sustainable system, which can automatically generate and render highly realistic virtual environments from data imported from available data sources like geographic information services (GIS) [9], [10]. Fig. 4 illustrates, how corresponding data and matching rendering modules, which integrate effects like realistic ground vegetation or a dynamic sky with clouds and weather effects can drastically improve the appearance and the degree of realism of virtual testing environments.

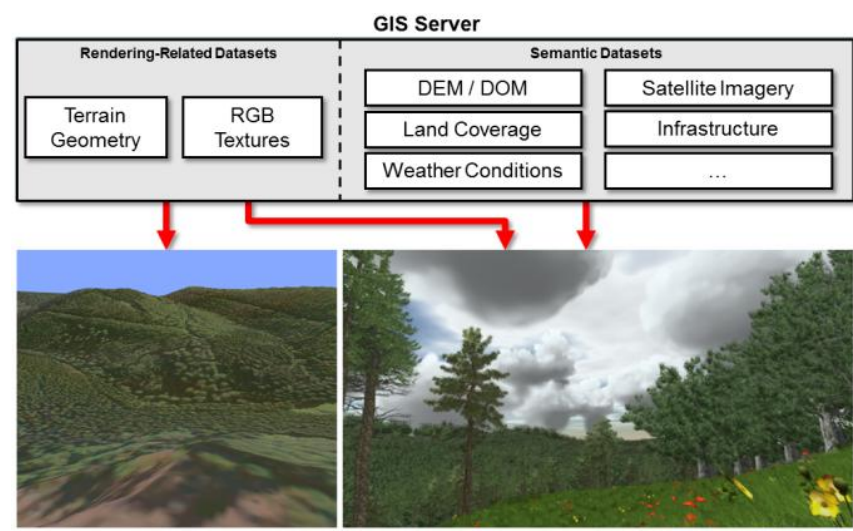

Fig. 4. Semantic data provided by GIS servers can be used to generate and render close-to-reality virtual mappings of the real environment with little effort.

However, eRobotics applications are not only aimed at improving the visual quality in engineering domains. They also aim to allow for accurate virtual testing of newly developed approaches and systems under realistic conditions. The VEROSIM Simulation System, which is co-developed at our institute in cooperation with our industrial partners, implements this system structure, does not only feature a state-of-the-art render framework, it also enables for the close interplay of all modules involved to enable rendering-supported simulations, e.g. in sensor-related domains.

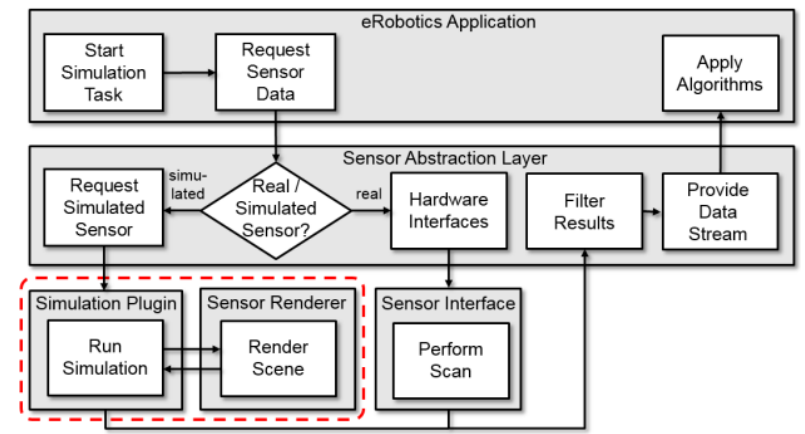

Fig. 5. General architecture for rendering-supported sensor simulations.

\section{RENDERING-SUPPORTED SENSOR SIMULATION}

As illustrated in Fig. 5, an application can request a sensor data stream through a sensor abstraction layer, which triggers a simulated or a real world sensor and provides the acquired data to the application to decouple application and sensor simulation development. In an ideal case, the application performs similarly with the simulated and the real world data. As illustrated by the red dotted box in the diagram, the sensor simulation consists of a simulation plugin, which can apply rendering techniques and data provided by the render framework in order to achieve improved accuracy and performance compared to common CPU-based approaches.

A practical example is demonstrated in Fig. 6, which shows the simulation of laser rays as emitted by LiDAR scanners in order to measure the distance to an object. As is 
the case of many optical sensors, the measurement accuracy directly depends on the reflectiveness of the hit surface. An important property that is given for all scanners is the minimum remission value (typically between $2 \%$ and $10 \%$ ), which defines the amount of emitted energy that need to be reflected back to the sensor for reliable measurements.

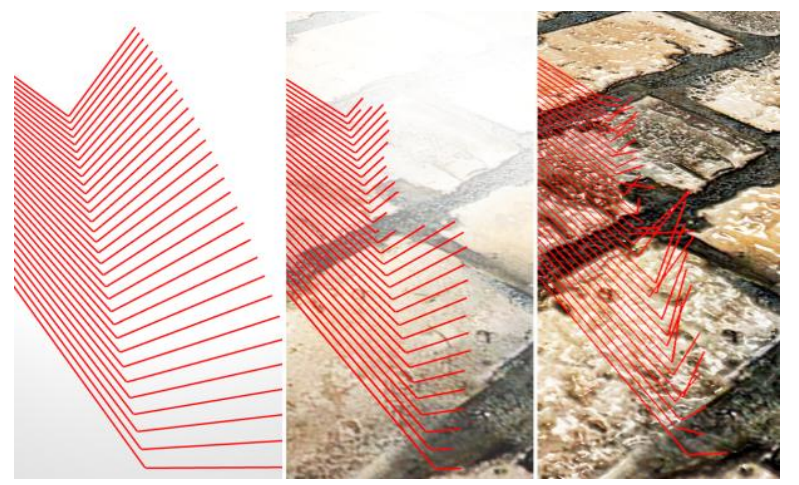

Fig. 6. Illustration of simulated LiDAR laser ray reflections without (left) and with the help of rendering data like textures (middle) and rendering approaches like normal mapping (right).

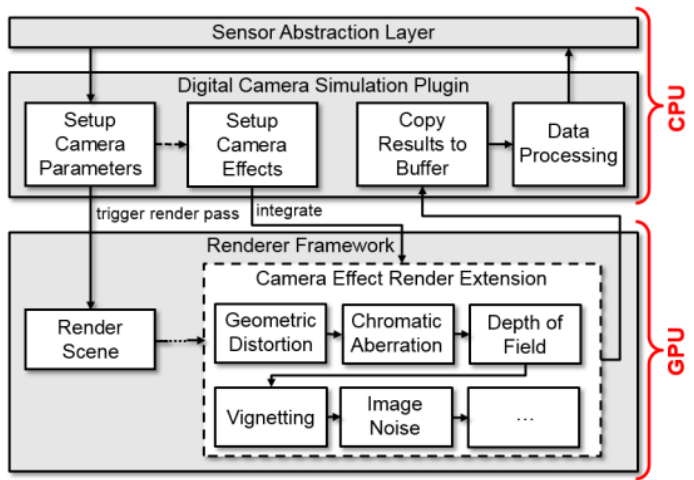

Fig. 7. Structure of the rendering-supported digital camera simulation, which considers typical sensor effects.

With common, CPU-based intersection tests, the material properties are defined globally for a polygon, which results in similar reflection intensities for the whole surface; however the renderer has much more information on the surface in order to render them more realistically. This rendering-related data, i.e. different kinds of textures, can directly be used to determine the reflectiveness for the simulated laser rays; additionally, commonly applied rendering techniques like normal mapping, specular mapping or displacement mapping further increase the accuracy of the simulation results with almost no additional effort since related data and techniques are usually available for rendering purposes.

Further rendering-supported sensor simulations have been implemented. While common digital camera simulations directly use the rendered images as input to computer vision or pattern matching algorithms, images acquired from real world cameras differ from ideal results due to technical and physical limitations. Digital cameras show effects like geometric distortion, chromatic aberration, image noise, etc., which can have a significant impact on the results achieved by applied CV approaches; hence, these effects need to be considered in the rendered images accordingly.

The simulation includes optical features like geometric distortion, chromatic aberration, depth-of-field and vignetting as well as sensor features like image noise and light bleeding, which can be simulated by using high dynamic range (HDR) rendering techniques. As illustrated in Fig. 7, these effects can be integrated into the rendering pipeline as a fully configurable post-process, which applies the named effects to the rendered images. After the pipeline execution, the image is optionally filtered and finally provided to the application. Fig. 8 compares a real world image taken by the Bumblebee XB3 stereo camera with the achieved simulation results.

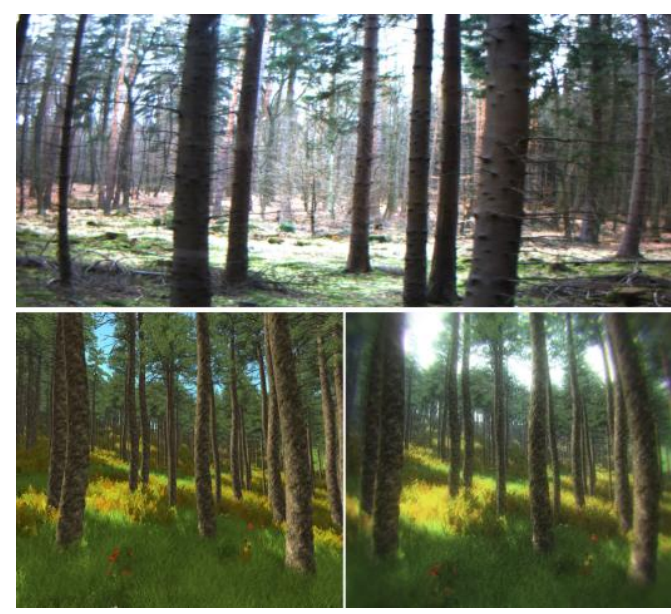

Fig. 8. Comparison of an image taken with the Bumblebee XB3 stereo camera (top) and the rendered image without (bottom left) and with (bottom right) the simulation of typical camera effects.

The same concept implies to the time-of-flight camera simulation shown in Fig. 9. First, geometric distortion for the acquired intensity image is simulated similarly to the digital camera simulation. Second, bilinear and jittered sampling between the pixel regions is used to properly simulate flying pixels and to produce more irregular results, which better match the wiggling behavior of real world sensors. Finally, systematic and material-dependent statistical noise is added. Real world ToF sensors usually filter the generated data to minimize the effects of the named errors before they provide them to the application. Similar filters routines are implemented in a second post-processing pipeline to provide results comparable to real world sensors.

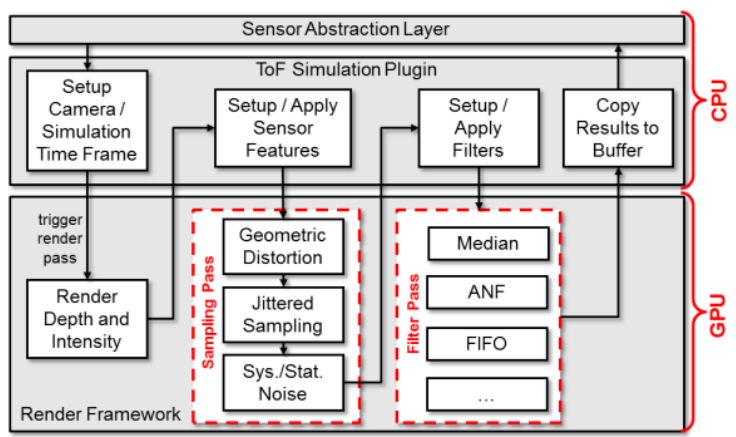

Fig. 9. Structure of the rendering-supported time-of-flight sensor simulation plugin.

While simple ToF sensor simulations sample the scene's depth information and directly generate an ideal point cloud, the developed GPU-based simulation pipeline is capable of simulating the whole processing chain with matching error models. In contrast to normal distributed statistical noise, the 
statistical noise of ToF images tend to look like a nearly periodic, sine-like function [7], which is also considered. Fig. 10 compares the results of simulated and real scanner data for a similar test scene. A solely geometry-based distance measurement approach without texture or intensity support would not be able to provide such realistic results in the shown scenario.

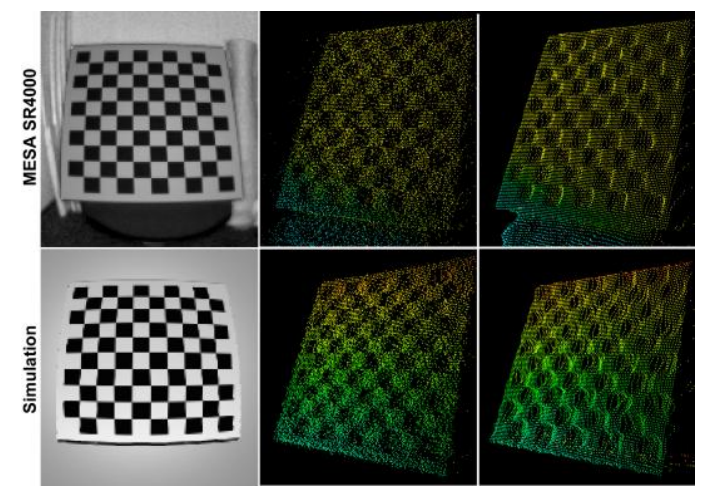

Fig. 10. Comparison of real world sensor data (top row) and results achieved with the presented simulation approaches (bottom row). Left: reflection intensity. Middle: unfiltered point cloud. Right: filtered point cloud.

\section{ApPlicAtions AND RESUlts}

A broad range of eRobotic applications have been realized that benefit from the presented sensor simulation approaches in combination with realistically rendered virtual environments [11]. An example in industrial robotic domains is shown in Fig. 11, where a 3D-LiDAR scanner was used to automatically generate and update a digital model for the real world robot workcell to apply dynamic path planning and workpiece handling strategies. The real world setup and scanning results in the top row show typical measurement errors (marked in red), which are caused by the highly reflective surface of the aluminum transport rails and the glossy parts of the robots that do not reflect enough light back to the sensor to measure the distance correctly. These errors are considered in the simulation shown in the bottom row, which allowes to make the developed approaches more robust before applying them to the real world setup.

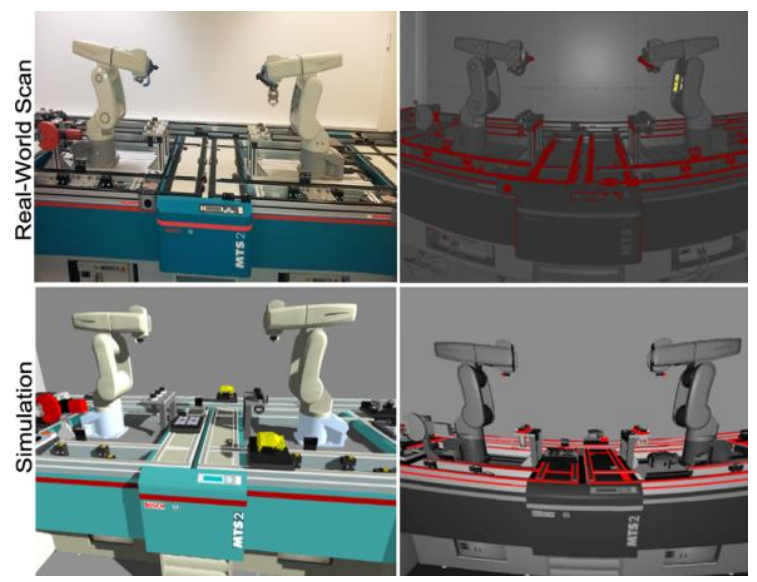

Fig. 11. Comparison of a real world and a simulated intensity image of a 3D LiDAR scan of a robot workcell.

Apart from industrial domains, many applications have been realized for mobile robotic domains in outdoor environments as part of the Virtual Forest project. As mentioned previously, data like satellite imagery, land coverage, aerial photos and digital elevation models as provided by GIS servers enable to (semi-)automatically generate a realistic virtual mapping of the real environment. These highly realistic virtual environments build the ideal test environment for close-to-reality testing and development of navigation and mapping approaches for mobile robots.

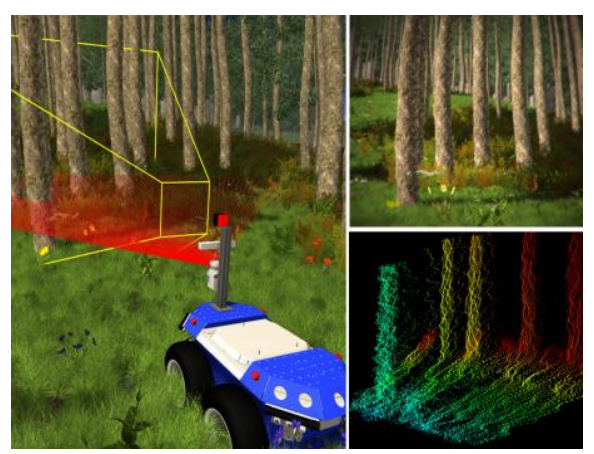

Fig. 12. Presented sensor simulations applied to the SeekurJr mobile robot Virtual Testbed in a simulated forest environment.

Fig. 12 shows a simulation of the SeekurJr mobile robot equipped with a stereo camera, a LiDAR scanner and a ToF camera, which is currently used as a testing platform for the virtual development and testing of navigation and mapping approaches. All presented sensor simulations are implemented as separate plugins that provide easily configurable sensor objects for convenient usage by the user, which allow for an easy transfer of previously developed components to novel projects or domains. Such a project is shown in Fig. 13 that demonstrates the aspects of knowledge transfer as well as the possibilities of early, visually attractive presentations of novel ideas and first results with low effort.

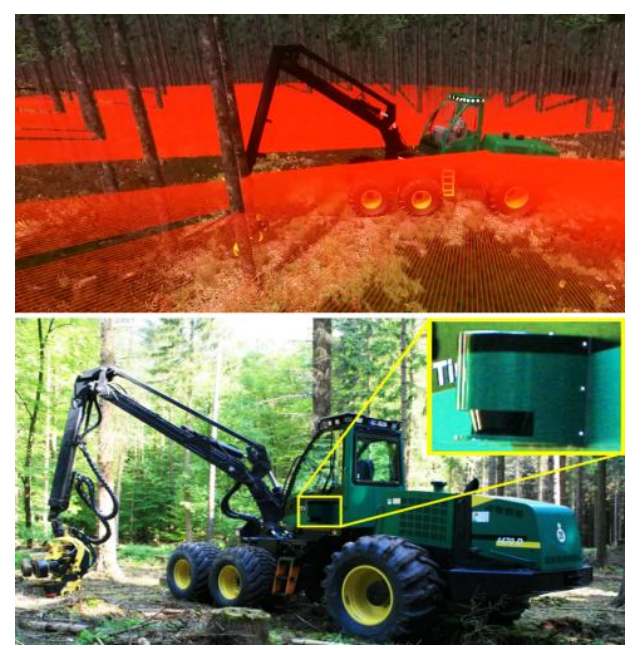

Fig. 13. Presented sensor simulations, which are applied to illustrate and test novel project ideas and virtual prototypes in attractive virtual environments.

LiDAR scanners are mounted on a wood harvester to support the machine operator by guiding him to selected trees in the stand marked for felling in order to improve the wood harvesting effectiveness. Due to the usage of the available virtual forest models, the simulated laser range scanners and the navigation and mapping algorithms developed for the presented SeekurJr mobile robot simulation, a fully functional demonstrator of such a harvester was created 
within a short period of time and with little effort. This demonstrator was further improved during the project instead of starting "from the scratch".

\section{CONCLUSION AND FUtURE WORK}

In this contribution, we presented the novel eRobotics approach, which aims to provide a comprehensive engineering software environment to realize complex robotics-related simulations with realistic VR environments. Apart from the visual enhancements provided by a state-of-the-art render framework, the interconnected system components allow for active rendering components that directly support essential simulation tasks like sensor simulations in order to achieve close-to-reality results that provide new possibilities for the development of novel robotics-related concepts. We also showed that eRobotics applications enable the convenient transfer of know-how and system components gathered from past projects to new ones, allowing for early results and high quality project demonstrators while similarly decreasing development time and project costs. Our future work will concentrate on the investigation of further rendering-supported sensor simulations, like radar-based or infrared sensors, as well as interdisciplinary areas like CFD simulations and data visualization to extend the domains addressed by eRobotics.

\section{REFERENCES}

[1] J. Roßmann, "The virtual testbed: Latest virtual reality technologies for space robotic applications," in Proc. the iSAIRAS 2008, pp. 1-8, Hollywood, USA, 2008.

[2] B. Balaguer, S. Balakirsky, S. Carpin, M. Lewis, and C. Scrapper, "USARSim: A validated simulator for research in robotics and automation," Workshop on Robot Simulators: Available Software, Scientific Applications, and Future Trends IEEE/RSJ, 2008.

[3] N. Keonig and A. Howard, "Design and use paradigms for gazebo, an open-source multi-robot simulator," in Proc. IEEE/RSJ International Conference on Intelligent Robots and Systems IROS, vol. 3, pp. 2149-2154, 2004.

[4] J. Sanders and E. Kandrot, CUDA by Example: An Introduction to General-Purpose GPU Programming, Addison-Wesley Longman, Amsterdam, 1st edition, 2010.

[5] M. Kucis and P. Zemcik, "Simulation of camera features," in Proc. the 16th Central European Seminar on Computer Graphics CESCG 2012, pp. 117-123, Smolenice, Slovakia, 2012.

[6] J. Rossmann, N. Hempe and M. Emde, "New methods of render-supported sensor simulation in modern real-time VR-simulation systems," in Proc. the 15th WSEAS CSCC Multiconference, pp. 358-364, Corfu, Greece, 2011.

[7] A. Kolb, E. Barth, R. Koch, and R. Larsen, "Time-of-flight cameras in computer graphics," Computer Graphics Forum, vol. 29, no. 1, pp. 141-159, 2010.

[8] J. Rossmann, "eRobotics: The symbiosis of advanced robotics and virtual reality technologies," in Proc. the 32nd ASME Computers and Information in Engineering Conference CIE 2012, vol. 2, pp. 1395-1403, Chicago, USA, 2012.

[9] N. Hempe and J. Rossmann, "A semantics-based, active render framework to realize complex eRobotics applications with realistic virtual testing environments," in Proc. the 7th European Modeling Symposium on Computer Modelling and Simulation EMS 2013, pp. 733-738, Pisa, Italy, 2013.

[10] N. Hempe and J. Roßmann, "Realizing the eRobotics approach in semantics-based multi-domain VR simulation systems," International Journal of Simulation Systems, Science \& Technology IJSSST, vol. 15 no. 2, pp. 98-108, 2014.

[11] J. Rossmann, "Advanced and efficient space robotics development: The eRobotics approach," in Proc. the 12th Symposium on Advanced Space Technologies in Robotics and Automation ASTRA 2013, pp. 1-8, Noordwijk, The Netherlands, 2013.

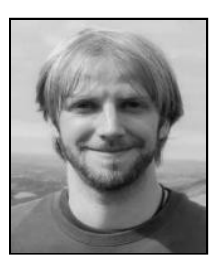

Nico Hempe studied computational visualistics at the University of Koblenz-Landau, Koblenz, Germany. He received his degree in computer science in 2006. Since 2006 he works as a researcher at the Institute for Man-Machine Interaction (MMI) at the RWTH Aachen University, Aachen, Germany.

His research interests include real-time computer graphics, virtual reality as well as space and industrial robotics. He focuses on VR simulation systems, data visualization and sensor simulation.

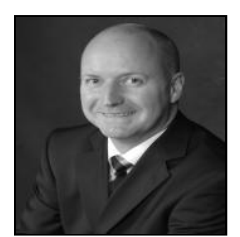

Jürgen Roßmann studied electrical engineering at the Universities of Dortmund and Bochum, Germany. After his studies, he worked as a researcher and team leader at the Institute of Robotics Research (IRF) in Dortmund.

He received his doctorate in 1993 and was a appointed visiting assistant professor for robotics and computer graphics at the University of Southern California in 1998. He received his habilitation degree in 2002 from the University of Dortmund and was managing director of EFR-systems GMBH in dortmund from 2005 to 2006.

Since 2006, he has been the director of the Institute for Man-Machine Interaction and a full professor at the RWTH Aachen University in Aachen, Germany. His research interests are projective virtual reality, multi-agent control and supervision, multi-sensor integration, system simulation and optimization techniques, computer vision, real-time visualization and man-machine interaction. 